\title{
Non-Typhoidal Salmonella Meningitis in an Adult Patient with HIV Infection: The Hostage-Taking Situation. Is There Any Solution?
}

\author{
Tarik Hanafi*, Hicham Titou, Hasna Kerrouch, Rachid Frikh, Naoufal Hjira and Mohammed Boui \\ Department of dermatology and venereology, Mohammed V Military Hospital, Faculty of Medicine and Pharmacy of \\ Rabat, Mohammed V University, Morocco \\ *Corresponding author: Tarik Hanafi, Department of dermatology and venereology, Mohammed $V$ \\ Military Hospital, Faculty of Medicine and Pharmacy of Rabat, Mohammed V University, Hay Riad 10000 \\ Rabat, Morocco
}

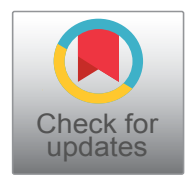

\begin{abstract}
We report a case of Non-Typhoidal Salmonella meningitis (NTS) in adult HIV patient with many relapses despite well-conducted treatment, we compare this situation to a hostage-taking situation, perpetrated by NTS, taking the body of HIV patient as a hostage who cannot get rid of this germ. This is a clinical alert to discuss the efficiency of prolonged oral administration of Trimethoprim/Sulfamethoxazole in preventing relapse and a whistleblower for the urgency of developing a vaccine.
\end{abstract}

\section{Keywords}

Non-Typhoidal salmonella meningitis, HIV, Relapse, Trimethoprim/Sulfamethoxazole, Vaccine

\section{Introduction}

Invasive strains of non-typhoidal salmonellae (NTS) have emerged as a prominent cause of bloodstream infection in African adults. The most important risk factor is HIV infection [1]. Adult HIV-positive have a markedly increased risk of contracting salmonellosis, which progresses to severe complications such as meningitis despite antimicrobial treatment [2]. Indeed, Bacterial meningitis is a medical, neurologic, and sometimes neurosurgical emergency that requires a multidisciplinary approach [3]. It is even more serious to manage when it occurs on immunocompromised field. We report a case of NTS meningitis in adult HIV patient with many relapses despite well-conducted treatment, we compare this situation to a hostage-taking situation, perpetrated by NTS, taking the body of HIV patient as a hostage who cannot get rid of this germ. This is a clinical alert to discuss the urgency of developing a vaccine.

\section{Case Report}

A 40-year-old man was admitted to our hospital because of Two-day history of fever, severe headache, diarrhea and acute altered mental status, physical examination finds a feverish patient at 39 Celsius, with a blood pressure of $10 / 06 \mathrm{mmHg}$, tachycardia at 108 beats per minute, respiratory rate at 28 cycles per minutes, altered consciousness with Glasgow coma scale $(\mathrm{GCS})=10$, and a stiff neck, Computerized Tomography (CT) scan showed no intracranial Abnormality, a lumbar puncture was performed, Empiric antibiotic treatment with intravenous (IV) ceftriaxone was Administered $2 \mathrm{~g} / 12 \mathrm{~h}$ pending results of the lumbar puncture, cerebrospinal fluid (CSF) was turbid, with low glucose $<0.05 \mathrm{~g} / \mathrm{l}$, low chloride: 112 $\mathrm{mmol} / \mathrm{l}$ and high protein: $3.31 \mathrm{~g} / \mathrm{l}$, Red cells: 450/ $\mathrm{mm}^{3}$, leukocytes: $1000 / \mathrm{mm}^{3}$ (neutrophils $75 \%$ Lymphocytes $25 \%$ ), CSF culture was positive for salmonella species typhimurium, antibiotic was administered for two weeks with sterilization of the lumbar puncture, gradually his fever decreased and his mental status improved, An Eliza reaction for HIV-1 was positive confirmed by a western blot, CD4/ cell count was $138 / \mathrm{mm}^{3}$ and viral load was 72056 copies per milliliter (copies $/ \mathrm{mL}$ ). We started antiretroviral therapy with ATENEF, one tablet per day, (TENOFOVIR DISOPROXIL FUMARATE 300 MG, EMTRICITABINE 200 MG,

Citation: Hanafi T, Titou H, Kerrouch H, Frikh R, Hjira N, et al. (2020) Non-Typhoidal Salmonella Meningitis in an Adult Patient with HIV Infection: The Hostage-Taking Situation. Is There Any Solution?. Clin Med Rev Case Rep 7:294. doi.org/10.23937/2378-3656/1410294

Accepted: January 23, 2020: Published: January 25, 2020

Copyright: (C) 2020 Hanafi T, et al. This is an open-access article distributed under the terms of the Creative Commons Attribution License, which permits unrestricted use, distribution, and reproduction in any medium, provided the original author and source are credited. 
EFAVIRENZ $600 \mathrm{MG}$ ). The patient remained afebrile for two weeks after stopping the antibiotic therapy, suddenly, he made a first relapse, he presented fever at $40 \mathrm{C}$, with severe headache and disturbances of consciousness, CSF was positive for salmonella spp, he was treated with ceftriaxone, this time used for four weeks with good evolution, three weeks after stopping the antibiotic therapy, he presented a second relapse, which was treated this time with ceftriaxone for four weeks followed by Trimethoprim/Sulfamethoxazole TMP-SMX $20 \mathrm{mg}(\mathrm{TMP}) / \mathrm{kg} / \mathrm{J}$ for three months, the patient did not relapse during a two-year follow-up.

\section{Discussion}

Invasive and recurrent salmonella infections are recognized as pathologies defining the AIDS stage in HIV-positive patients [4]. Studies of NTS meningitis in HIV-positive patients have been limited and Focal complications have rarely been reported, in a Spanish study, Ten of 38 HIV-infected patients with salmonellosis documented over a period of 9 years had focal suppurative complications [5], it's even rarer for meningeal localization, with only one case out of ten patients, who presented with salmonella Choleraesuis meningitis with CD4 lymphocyte count $=162 / \mathrm{mm}^{3}$, one year before admission, the patient died 24 hours after admission to a septic shock, despite antibiotic treatment with combination of cefotaxime and ampicillin. In 2011, a literature review proposed by Belloso, et al. [6] had only found ten cases of NTS meningitis reported in the literature, incriminated serotypes were: Salmonella Enteritidis (4), Typhimurium (2), Choleraesuis (1), Heidelberg (1), Group D (1) and Infantis (1). In Africa, we found only one series [7], reported in South Africa with 62 cases of NTS meningitis in HIV positive patients, 32 cases with salmonella typhimurium, and only 45 cases over 15 -years-old, this study concluded that NTS meningitis in South Africa is highly associated with HIV in adults, and with Salmonella Typhimurium ST313.

The mortality rate is high $=50 \%$ [6], and altered mental status was described as a risk factor for mortality $[7,8]$, that was the case in our patient with a GCS at 10 , without leading to the death probably thanks to early antibiotic therapy.

Relapses were observed in 64\% [2], in some cases, up to five times, in only three months for the same patient [6], in our case we noted only two relapses, These relapses compared in our study to a hostage-taking situation are explained by recent pathogenic elements, indeed, three key defects [1] that contribute to the pathogenesis of invasive non-typhoidal salmonellae in HIV-positive people have been recently described, that include the Loss of interleukin 17-producing CD4 T cells from the gut mucosa in HIV, an Humoral defect in HIV and the third key which is most interesting for our case, is the Cellular cytokine dysregulation and attenuation of proinflammatory responses during intracellular co-infection with HIV and invasive non-typhoidal salmonellae which leads to, intracellular sanctuary site of persistence, Salmonella can escape killing by these phagocytes and survive and multiply within them, giving rise to chronic infections [9].

Therapeutic failures are usually associated with persistence and incomplete clearance of the infection, the recommended treatment is third generation cephalosporins, with or without a fluoroquinolone over a prolonged period up to four weeks given the risk of relapse [10].

In cases where these drugs cannot be afforded, cotrimoxazole may be considered. In our case we believe that the oral administration of TMP-SMX extend over three months, was efficient in preventing relapse as has been mentioned by some authors [11].

Despite the limited number of cases in the literature, we think that NTS meningitis in HIV-positive African patients is underdiagnosed, especially in resource-poor countries with high rates of HIV and lack of laboratories. We really believe that There is an urgent need for development and implementation of vaccine $[1,12]$.

\section{Conclusion}

This is a clinical alert to discuss the efficiency of prolonged oral administration of Trimethoprim/ Sulfamethoxazole in preventing relapse and a whistleblower for the urgency of developing a vaccine. Further studies would be required to demonstrate whether our patient was actually released from NTS by the association of Trimethoprim/Sulfamethoxazole.

\section{References}

1. Nicholas A Feasey, Gordon Dougan, Robert A Kingsley, Robert S Heyderman, Melita A (2012) Invasive non-typhoidal salmonella disease: An emerging and neglected tropical disease in Africa Gordon. Lancet 379: 2489-2499.

2. Subramoney EL (2015) Non-typhoidal Salmonella infections in HIV-positive adults. South African Medical Journal 105: 805-807.

3. Van de Beek D, de Gans J, Tunkel AR, Wijdicks EF (2006) Community-acquired bacterial meningitis in adults. $\mathrm{N}$ Engl J Med 354: 44-53.

4. Benson CA, Kaplan JE, Masur H, Pau A, Holmes KK (2004) Treating opportunistic infections among HIV-infected adults and adolescents. MMWR 53: 1-112.

5. Fernández Guerrero ML, Ramos JM, Núñez A, Cuenca M, de Górgolas M (1997) Focal infections due to non-typhi Salmonella in patients with AIDS: Report of 10 cases and review. Clin Infect Dis 25: 690-697.

6. Belloso WH, Romano M, Greco GS, Davey RT, Perelsztein AG, et al. (2011) Recurrent meningitis and subarachnoid 
hemorrhage due to salmonella in an HIV+ Patient: Case report and mini review of the literature. Open AIDS J 5: 62-66.

7. Keddy KH, Sooka A, Musekiwa A, Smith AM, Ismail H, et al (2015) Clinical and microbiological features of salmonella meningitis in a South African Population, 2003-2013. Clin Infect Dis 61: S272-S282.

8. Leonard MK, Murrow JR, Jurado R, Gaynes R (2002) Salmonella meningitis in adults infected with HIV: Case report and review of the literature. Am J Med Sci 323: 266-268.

9. Thomas Ruby, Laura McLaughlin, Smita Gopinath, Denise Monack (2012) Salmonella's long-term relationship with its host. FEMS Microbiol Rev 36: 600-615.
10. Alex Owusu-Ofori, W. Michael Scheld (2003) Treatment of Salmonella meningitis: Two case reports and a review of the literature. Int J Infect Dis 7: 53-60.

11. Murphy TF, Fernald GW (1983) Trimethoprim-sulfamethoxazole therapy for relapses of Salmonella meningitis. Pediatr Infect Dis 2: 465-468.

12. Tennant SM, MacLennan CA, Simon R, Martin LB, Khan MI (2016) Nontyphoidal salmonella disease: Current status of vaccine research and development. Vaccine 34: 29072910. 